\title{
Different responses of the GlnB and GlnZ proteins upon in vitro uridylylation by the Azospirillum brasilense GlnD protein
}

\author{
L.M. Araújo, L.F. Huergo, A.L. Invitti, C.I. Gimenes, A.C. Bonatto, R.A. Monteiro, \\ E.M. Souza, F.O. Pedrosa and L.S. Chubatsu
}

Departamento de Bioquímica e Biologia Molecular, Universidade Federal do Paraná, Curitiba, PR, Brasil

Correspondence to: L.S. Chubatsu, Departamento de Bioquímica e Biologia Molecular, Universidade

Federal do Paraná, Caixa Postal 19046, 81531-990 Curitiba, PR, Brasil

Fax: +55-41-3266-2042. E-mail: chubatsu@ufpr.br

\begin{abstract}
Azospirillum brasilense is a diazotroph found in association with important agricultural crops. In this organism, the regulation of nitrogen fixation by ammonium ions involves several proteins including the uridylyltransferase/uridylyl-removing enzyme, GInD, which reversibly uridylylates the two PII proteins, GInB and GlnZ, in response to the concentration of ammonium ions. In the present study, the uridylylation/deuridylylation cycle of $A$. brasilense GlnB and GlnZ proteins by GlnD was reconstituted in vitro using the purified proteins. The uridylylation assay was analyzed using non-denaturing polyacrylamide gel electrophoresis and fluorescent protein detection. Our results show that the purified $A$. brasilense GlnB and GlnZ proteins were uridylylated by the purified $A$. brasilense GInD protein in a process dependent on ATP and 2-oxoglutarate. The dependence on ATP for uridylylation was similar for both proteins. On the other hand, at micromolar concentration of 2-oxoglutarate (up to $100 \mu \mathrm{M}$ ), GInB uridylylation was almost twice that of GlnZ, an effect that was not observed at higher concentrations of 2-oxoglutarate (up to $10 \mathrm{mM}$ ). Glutamine inhibited uridylylation and stimulated deuridylylation of both GInB and GInZ. However, glutamine seemed to inhibit GlnZ uridylylation more efficiently. Our results suggest that the differences in the uridylylation pattern of GInB and GlnZ might be important for fine-tuning of the signaling pathway of cellular nitrogen status in A. brasilense.
\end{abstract}

Key words: Azospirillum brasilense; Nitrogen fixation; PII-like protein; GInD; GlnB; GlnZ

Research supported by CAPES, CNPq, CNPq/PIBIC, MCT/CNPq/Institutos do Milênio, Fundação Araucária, and Paraná Tecnologia.

Received August 16, 2007. Accepted February 29, 2008

Proteins from the PII family comprise a class of highly conserved regulatory proteins which are among the most widely distributed in nature. In Gram-negative bacteria, these proteins are homotrimers of about $12 \mathrm{kDa}$ per monomer, and each subunit can be reversibly modified by uridylylation at the tyrosine 51 residue in response to fluctuation in the availably of ammonium ion in the external medium (1). The reversible uridylylation of PII is catalyzed by the GlnD protein, a bifunctional uridylyltransferase/uridylyl-removing enzyme (2).

When the external ammonium ion supply is low, the intracellular glutamine concentration is low, favoring the promotion of PII uridylylation by the uridylyltransferase activity of GInD. Upon an ammonium ion increase, the intracellular glutamine concentration increases, triggering the uridylyl-removing enzyme activity of GInD and leading to PII deuridylylation $(3,4)$. Whether unmodified or uridylylated, the PII proteins signal high and low ammonium ion concentration, respectively, to specific regulatory target proteins such as AmtB, NtrB, and ATase $(1,4)$, controlling their activities by protein-protein interactions. In addition to posttranslational modification, binding of small regulatory effectors such as adenine nucleotides and 2-oxoglutarate can also control PII protein activity (1).

Azospirillum brasilense, a diazotrophic $\alpha$-proteobacterium associated with important agricultural crops (5), has 
two genes coding for PII-like proteins, $g \ln B$ and $g \ln Z(6)$, which are predominantly expressed under nitrogen-limiting conditions $(6,7)$. Their role in nitrogen fixation by $A$. brasilense has been studied in several laboratories and it has been shown that GlnB is absolutely required for NifA activity $(8,9)$ and that both $G \ln B$ and $G \ln Z$ are involved in posttranslational control of nitrogenase $(10,11)$.

In order to characterize the function of $\mathrm{G} \ln \mathrm{D}, \mathrm{G} \ln \mathrm{B}$, and GlnZ in signaling the external ammonium ion levels in $A$. brasilense, we analyzed the in vitro uridylylation of GlnB and GInZ catalyzed by the GInD protein.

For over-expression of $\mathrm{G} \ln D$, the $g \ln D$ gene of $A$. brasilense strain FP2 was amplified using purified genomic DNA as a template and primers based on the sequence deposited in the GenBank database (Accession No. AF149716). Primers AbglnDN (ACGTTTTGCATATGCT CTCCAC) and AbglnDC (CTGTCCTGGATCCCGGCTTT) were aligned to the 5 ' and 3 ' ends, respectively, of the $g / n D$ coding sequence with $\mathrm{Ndel}$ and $\mathrm{BamHI}$ sites (underlined) introduced in the amplified fragment in order to facilitate cloning. This fragment was cloned into the pET28a vector (Novagen, USA), yielding the pALIpGT plasmid. The cloned fragment was completely sequenced to confirm its integrity. The $A$. brasilense GInD protein was over-expressed as a His-tag protein (GlnD-His) in the Escherichia coli glnBglnD- strain RB9065( $\lambda$ DE3) upon induction with 0.5 $\mathrm{mM}$ isopropyl ß-D-thiogalactopyranoside (IPTG) at $37^{\circ} \mathrm{C}$ for $3 \mathrm{~h}$. Cells were harvested, resuspended in buffer $\mathrm{S}$ (50 $\mathrm{mM}$ Tris- $\mathrm{HCl}, \mathrm{pH} 8.0,500 \mathrm{mM} \mathrm{NaCl}, 1 \mathrm{mM}$ dithiothreitol, $10 \%$ glycerol) and then lysed by sonication. The crude extract was then centrifuged at 10,000 rpm for $15 \mathrm{~min}$ and the supernatant was loaded onto a Hi-trap-chelating-Ni ${ }^{2+}$ column (GE Healthcare, USA) pre-equilibrated in buffer $\mathrm{S}$. Two washing steps were performed using ten column volumes each of buffer $\mathrm{W}(50 \mathrm{mM}$ Tris- $\mathrm{HCl}, \mathrm{pH} 6.3,500$ $\mathrm{mM} \mathrm{NaCl}$ ) with 10 and $40 \mathrm{mM}$ imidazole. The proteins were eluted by a stepwise increase of imidazole (50, 100, 300, and $500 \mathrm{mM}$ ) in buffer containing $50 \mathrm{mM}$ Tris- $\mathrm{HCl}, \mathrm{pH} 8.0$, $100 \mathrm{mM} \mathrm{KCl}$, and $10 \%$ glycerol, using five column volumes at each concentration. Fractions were analyzed by sodium dodecyl sulfate-polyacrylamide gel electrophoresis (SDSPAGE) and those containing GInD-His with purity over $97 \%$, as determined by densitometric analysis, were stored at $-70^{\circ} \mathrm{C}$ and used in our assays.

The native $A$. brasilense $\mathrm{GlnB}$ and $\mathrm{GlnZ}$ proteins were

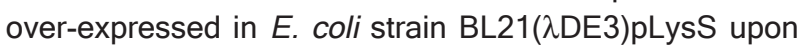
plasmids pLMA4 (9) and pMSA-L1 (12), respectively, and induction with $0.5 \mathrm{mM}$ IPTG at $37^{\circ} \mathrm{C}$ for $3 \mathrm{~h}$. After harvesting, cells were resuspended in buffer $\mathrm{SN}(50 \mathrm{mM}$ Tris- $\mathrm{HCl}$, $\mathrm{pH}$ 7.5, $200 \mathrm{mM} \mathrm{KCl}, 1 \mathrm{mM}$ EDTA, and 20\% glycerol) and lysed by sonication. The crude extract was clarified by centrifugation at $10,000 \mathrm{rpm}$ for $20 \mathrm{~min}$, and the soluble fraction was loaded onto a Hi-trap-heparin column (GE Healthcare) pre-equilibrated in buffer A (50 mM Tris- $\mathrm{HCl}$, $\mathrm{pH} 7.5,100 \mathrm{mM} \mathrm{KCl}$, and $1 \mathrm{mM}$ EDTA). After washing with five column volumes of buffer $A$, proteins were eluted by using a linear $\mathrm{KCl}$ gradient $(0.1$ to $1 \mathrm{mM})$ in buffer $A$. Fractions were analyzed by SDS-PAGE and purity was determined by densitometry. Our procedure efficiently purified the GInB and GInZ proteins using only one chromatographic step, reaching purity of $95 \%$ for GInB and of $92 \%$ for GInZ, as determined by densitometric analysis by SDSPAGE. These fractions were used in our assays. We observed that ion-exchange chromatography and/or protamine sulfate pre-treatment, used regularly as procedure for PII purification, also eliminated a significant amount of PII proteins and were not essential for $A$. brasilense GInB or GInZ purification (data not shown).

Although E. coli BL21( $2 D E 3) p L y s S$ can produce endogenous GInB and GInK, allowing the formation of heterotrimers (13) this is unlikely to be the case, since the expression of $E$. coli GInB is significantly low (14) compared to the high expression of the recombinant $A$. brasilense $\mathrm{GlnB}$ or $\mathrm{GlnZ}$, and there is no significant expression of $E$. coli GlnK in the N-rich medium used for protein induction. Also, the purified GInB and GlnZ migrated as sharp and homogenous bands by native gel electrophoresis, suggesting that most of the purified proteins were nonmodified homotrimers (Figure 1A).

The uridylylation assay was performed as described (12) and analyzed by non-denaturing PAGE (Figure 1A). Densitometric analyses of SYPRO-Red-stained (Invitrogen, USA) gels were performed to determine the percentage of PII uridylylation. Given the trimeric nature of all PII proteins studied so far, we expected to observe up to four forms of these proteins: non-uridylylated PII, PII-UMP, PII$\mathrm{UMP}_{2}$, and $\mathrm{PII}_{\mathrm{U}} \mathrm{UMP}_{3}$ on native gels.

The effect of GlnD concentration on the uridylylation of purified GInB and GlnZ is shown in Figure 1. During 30-min incubation, an increasing amount of purified GInD produced an increase in uridylylation of the PII subunits, achieving total uridylylation of $\mathrm{GlnB}$ and near $96 \%$ of $\mathrm{Gln} Z$ with $100 \mathrm{nM}$ of purified GInD (Figure 1A,B). With lower amounts of GlnD we observed all four possible forms of GlnZ in the gel (i.e., GlnZ, GlnZ-UMP 1 , GlnZ-UMP , and GInZ-UMP ${ }_{3}$ ) since, as expected, the addition of the negatively charged UMP group increased protein migration (Figure 1A). Interestingly, for GInB only two forms were observed on the gels (Figure $1 \mathrm{~A}$ ) and this result was reproduced in different experiments using different GInB preparations (data not shown).

To check the nature of the GlnB forms observed in the 
native gels (Figure $1 \mathrm{~A})$ we performed mass spectrometry analyses (Maldi-ToF) of the GlnB samples before and after the uridylylation reactions by mixing them with sinapic acid matrix (1:9), spotting on a Maldi plate (Bruker Daltonics, Bremen, Germany) and allowing the sample to dry. Mass spectra were acquired using a Maldi-ToF-MS Autoflex spectrometer (Bruker Daltonics) in a positive linear mode with an accelerating voltage of $20 \mathrm{kV}$, delay time of $330 \mathrm{~ns}$ and acquisition mass range of 5-20 kDa. Peak lists were created with the sophisticated numerical annotation procedure algorithm using the FlexAnalysis 2.0 software (Bruker Daltonics). Before incubation with GInD, the GlnB Maldi-ToF analyses yielded a single peak with an $\mathrm{m} / \mathrm{z}$ ratio of 12,367 , which was in good agreement with the predicted monomeric molecular mass of $12,371 \mathrm{kDa}$. After incubation with $100 \mathrm{nM}$ GInD, the GlnB Maldi-ToF analysis yielded a single peak with an $\mathrm{m} / \mathrm{z}$ ratio of 12,672 , corresponding to the GInB monomer covalently bound to UMP. Maldi-ToF analyses of incomplete uridylylation reactions yield two peaks with an $\mathrm{m} / \mathrm{z}$ ratio corresponding to the unmodified and modified forms of monomeric GlnB. These results suggest that the faster migrating band observed on the gel after incubation with $100 \mathrm{nM}$ GInD is the homogeneous trimeric modified form of GlnB, GlnB-UMP ${ }_{3}$.

It is unlikely that the absence of other GInB intermediates in the native gels (i.e., containing 1 or 2 UMP) is a result of co-migration of the mono-, di- and tri-uridylylated forms as a single band on the gels. As the migration profile on native gels depends on the protein charge and conformation, the superimposition of GlnB-UMP $1, G \operatorname{lnB}-U_{M P}$, and GInB-UMP ${ }_{3}$ as a single band would imply major conformational changes to counterbalance the effect of the presence of more negatively charged UMP groups. This scenario seems unlikely since the site of PII uridylylation (Tyr51) is located in the T-loop which seems very flexible and extends away from the core structure of the PII trimer (1).

The presence of only two GlnB forms was also observed in native gels when the $A$. brasilense GlnB was assayed for in vivo deuridylylation and re-uridylylation (11), suggesting that this could be the case not only for uridylylation but also for re-uridylylation.

The physiological role of the different pattern of GlnB modification as compared to GInZ is still unclear. Several known Pll-target interactions are controlled by the PII uridylylation status and such response might be necessary especially for a trimer-to-trimer interaction as that occurring with $A$. brasilense AmtB-GInB (15). The AmtB protein acts as a trimer containing three ammonia channels. Binding of a PII trimer to the cytoplasmic surface of AmtB inhibits channel activity because the non-uridylylated PII
T-loop from each monomer blocks one AmtB channel pore $(16,17)$. The partially modified GlnB (i.e., GInB-UMP 1 and GInB-UMP 2 ) could compete with fully deuridylylated GInB for AmtB binding but only the latter would effectively block channel activity. In this case a full or null interaction might be essential.

It has been shown that PII uridylylation is controlled by the effectors ATP and 2-oxoglutarate in many organisms $(1,3)$. To analyze the effect of these effectors on the uridylylation of $A$. brasilense PII proteins, reactions were conducted in the presence of varying concentrations of ATP and 2-oxoglutarate (Figure 2A,B). In the absence of ATP or 2-oxoglutarate, purified $A$. brasilense GInD was
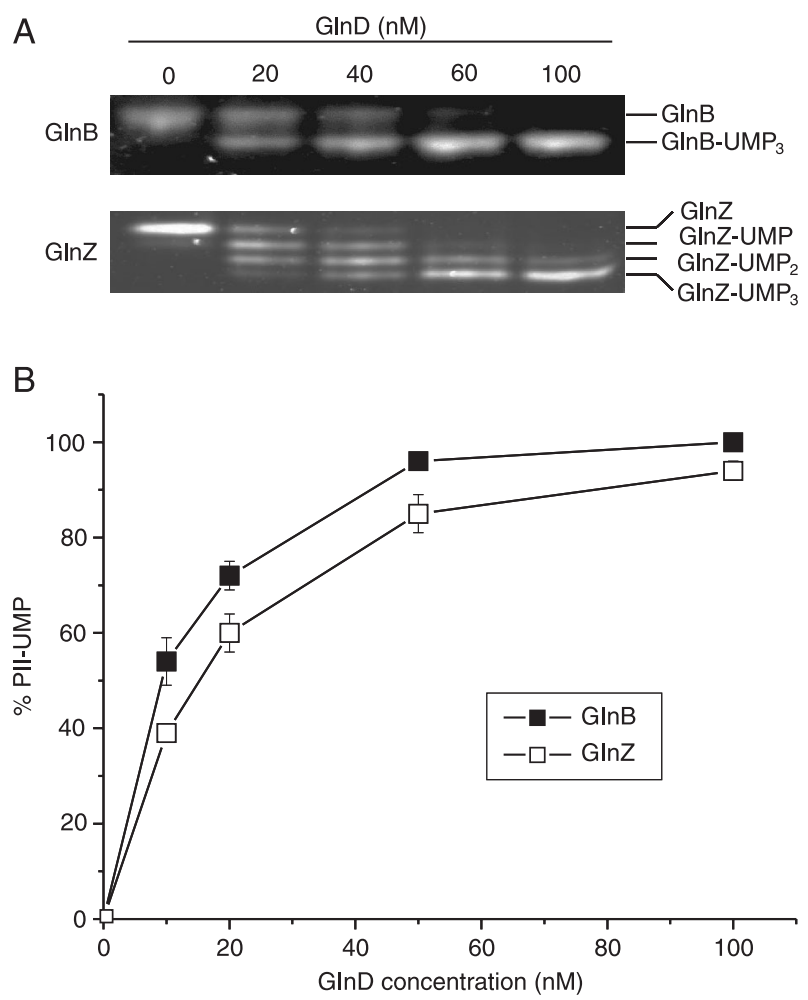

Figure 1. In vitro uridylylation of the $\mathrm{GlnB}$ and $\mathrm{GlnZ}$ proteins (PII) using Azospirillum brasilense GlnD. Reactions were performed in uridylylation buffer (100 mM Tris- $\mathrm{HCl}, \mathrm{pH} 7.5,100 \mathrm{mM} \mathrm{KCl}, 25$ $\mathrm{mM} \mathrm{MgCl} 2$, and $0.1 \mathrm{mM}$ dithiothreitol) using $3 \mu \mathrm{M}$ of each PII protein, $0.1 \mathrm{mM}$ ATP, $10 \mathrm{mM}$ 2-oxoglutarate, $1 \mathrm{mM}$ UTP, and the indicated concentration of GlnD. Samples were incubated for 30 $\min$ at $30^{\circ} \mathrm{C}$ and the reactions were stopped by adding $10 \mathrm{mM}$ EDTA and $5 \mu \mathrm{L}$ electrophoresis sample buffer $(0.5 \mathrm{mM}$ Tris-Cl, $\mathrm{pH} 6.8,10 \%$ glycerol, and $0.05 \%$ (w/v) bromophenol blue). Samples were kept on ice before loading for non-denaturing $10 \%$ polyacrylamide gel electrophoresis. Panel $A$, the gel shows a representative experiment stained with SYPRO Red. Lines indicate the unmodified and modified PII species. Panel B shows a densitometric analysis of at least three independent experiments. Data are reported as means \pm SD. 
unable to uridylylate the GInB or GInZ proteins (Figure $2 A, B)$. Maximum uridylylation was achieved with our purified GInB and GInZ at $0.1 \mathrm{mM} \mathrm{ATP} \mathrm{(Figure} 2 \mathrm{~A}$ ) and $1 \mathrm{mM}$ 2-oxoglutarate (Figure 2B). The requirement for ATP in the uridylylation reaction was similar for both proteins. On the other hand, the dependence on 2-oxoglutarate seems to be different for GlnB and GlnZ in the micromolar range. At $0.1 \mathrm{mM} 2$-oxoglutarate or less, the uridylylation of GlnB was considerably higher than that of GlnZ (Figure 2B). On the other hand, at millimolar concentrations of 2-oxoglutarate, a similar response was observed for both proteins. Although the intracellular concentration of 2-oxoglutarate in $A$. brasilense is unknown, in $E$. coli the levels of 2oxoglutarate are known to vary from 0.1 to $0.9 \mathrm{mM}$ (18), suggesting that the uridylylation state of $\mathrm{GlnB}$ and $\mathrm{Gln} Z$ may also translate the cell carbon status.

ATP and 2-oxoglutarate are required for the uridylylation of $A$. brasilense GInB and GInZ, probably by the binding of
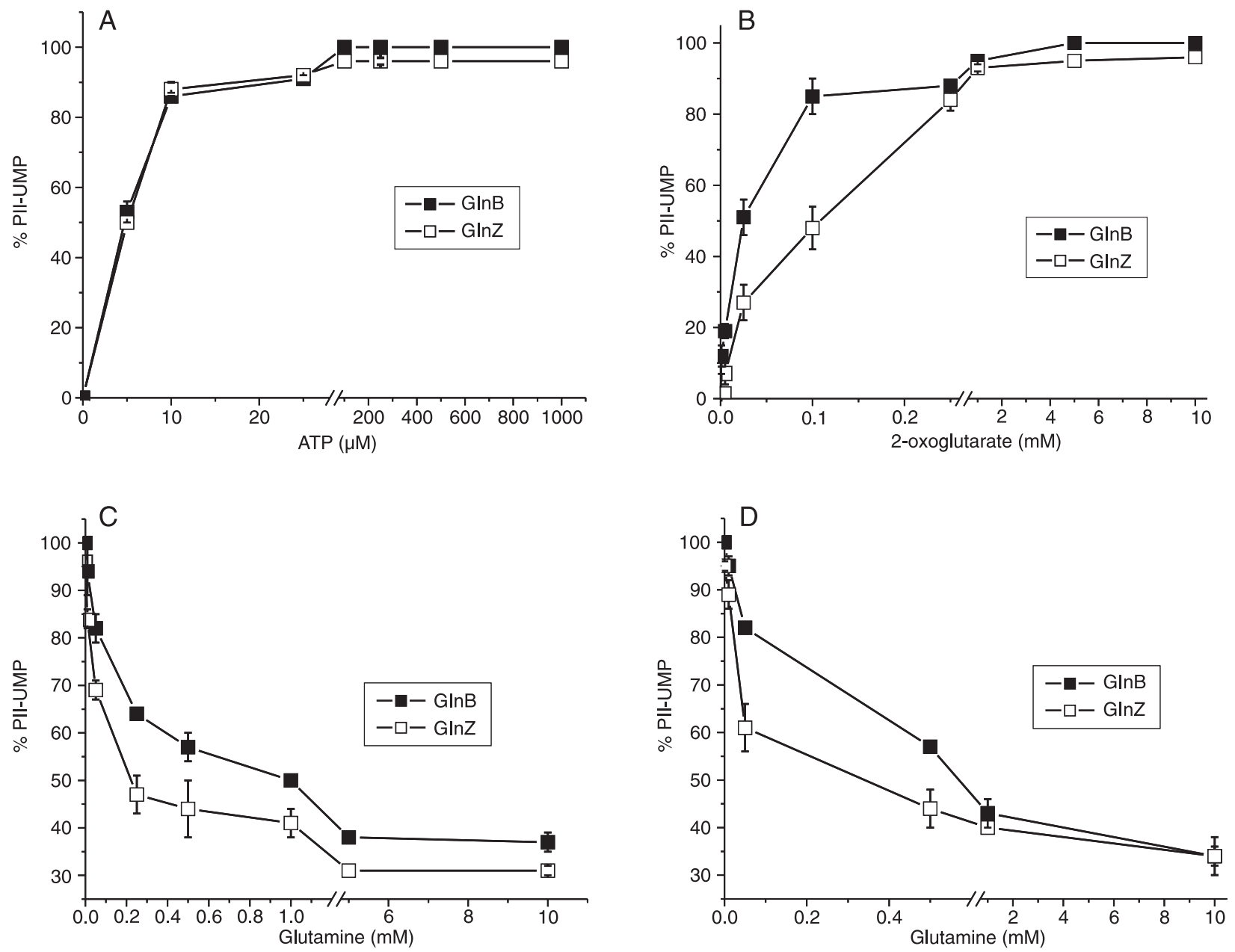

Figure 2. Effect of ATP (panel A), 2-oxoglutarate (panel B) and glutamine (panel C) on the in vitro uridylylation and the effect of glutamine on the in vitro deuridylylation (panel D) of purified Azospirillum brasilense GlnB and GlnZ proteins by purified A. brasilense GInD. For the uridylylation assay, reactions were carried out with $3 \mu \mathrm{M}$ PII proteins, $1 \mathrm{mM}$ UTP, $100 \mathrm{nM}$ GInD, and $0.1 \mathrm{mM}$ ATP (panels B and C) or $10 \mathrm{mM}$ 2-oxoglutarate (panels A and C). Samples were incubated for $30 \mathrm{~min}$ at $30^{\circ} \mathrm{C}$ and loaded for nondenaturing $10 \%$ polyacrylamide gel electrophoresis. For the deuridylylation assay (panel D), samples were first fully uridylylated for 30 min under optimum uridylylation conditions, and then loaded onto a gel filtration column (GS25, GE Healthcare) pre-equilibrated with uridylylation buffer. The elution was carried out using the same buffer, and the eluted GInB-UMP 3 and GlnZ-UMP 3 proteins were then used in the deuridylylation reaction. PII-UMP 3 proteins $(3 \mu \mathrm{M})$ were incubated with $1 \mathrm{mM}$ UTP, $2 \mathrm{mM}$ 2-oxoglutarate, $0.1 \mathrm{mM}$ ATP and $100 \mu \mathrm{M} \mathrm{GlnD}$ and the indicated concentrations of glutamine in uridylylation buffer for $30 \mathrm{~min}$ at $30^{\circ} \mathrm{C}$. Reactions were then loaded for non-denaturing $10 \%$ polyacrylamide gel electrophoresis. Densitometric analyses were performed with SYPRO red-stained gels and indicate the percentage of modified PII protein. Results show the average of at least two independent experiments. 
these effectors to the PII protein as described for E. coli PII (19). Glutamine is also a regulator of PII protein uridylylation. The activity of $E$. coli GInD is directly controlled by the levels of glutamine (20), which has a dual function: it inhibits the uridylyltransferase activity and stimulates the uridylyl-removing activity of $\operatorname{GInD}(7,20)$. We tested the effect of glutamine on the uridylylation and deuridylylation of GlnB and GInZ (Figure 2C,D). In the absence of glutamine, a situation indicating nitrogen deficiency, the $G \ln B$ and GInZ uridylylation rate occurs at its maximum level (Figure 2C). However, by increasing the amounts of glutamine in the reaction, an inhibition of uridylylation was observed. At $10 \mathrm{mM}$ glutamine, less than $40 \%$ uridylylation of GInB and GInZ occurred. This inhibition of uridylylation by glutamine seems to be higher for the $G \ln Z$ than the $G \ln B$ protein (Figure $2 \mathrm{C}$ ).

We also analyzed the deuridylylation of GInB-UMP and GInZ-UMP ${ }_{3}$ by purified $A$. brasilense $\mathrm{GInD}$ in response to the presence of glutamine (Figure 2D). At $1 \mathrm{mM}$ glutamine, both proteins were deuridylylated up to $65 \%$. Interestingly, at lower glutamine concentrations, the extent of deuridylylation of GlnZ-UMP 3 was higher than that of GlnB$\mathrm{UMP}_{3}$ (Figure 2D).

\section{References}

1. Arcondeguy $T$, Jack R, Merrick $M . P(I I)$ signal transduction proteins, pivotal players in microbial nitrogen control. Microbiol Mol Biol Rev 2001; 65: 80-105.

2. Adler SP, Purich D, Stadtman ER. Cascade control of Escherichia coli glutamine synthetase. Properties of the PII regulatory protein and the uridylyltransferase-uridylyl-removing enzyme. J Biol Chem 1975; 250: 6264-6272.

3. Ninfa AJ, Jiang P. PII signal transduction proteins: sensors of alpha-ketoglutarate that regulate nitrogen metabolism. Curr Opin Microbiol 2005; 8: 168-173.

4. Commichau FM, Forchhammer K, Stulke J. Regulatory links between carbon and nitrogen metabolism. Curr Opin Microbiol 2006; 9: 167-172.

5. Döbereiner J, Pedrosa FO. Nitrogen-fixing bacteria in nonleguminous crop plants. Madison: Springer-Verlag: Science Tech Publishers; 1987.

6. de Zamaroczy M. Structural homologues $P(I I)$ and $P(Z)$ of Azospirillum brasilense provide intracellular signalling for selective regulation of various nitrogen-dependent functions. Mol Microbiol 1998; 29: 449-463.

7. Huergo LF, Souza EM, Steffens MB, Yates MG, Pedrosa FO, Chubatsu LS. Regulation of glnB gene promoter expression in Azospirillum brasilense by the NtrC protein. FEMS Microbiol Lett 2003; 223: 33-40.

8. Arsene F, Kaminski PA, Elmerich C. Modulation of NifA activity by PII in Azospirillum brasilense: evidence for a regulatory role of the NifA $\mathrm{N}$-terminal domain. J Bacteriol
Furthermore, partial deuridylylation of $G \ln Z$ resulted in up to 4 bands representing the $G \operatorname{lnZ}(U M P), G \ln Z(U M P)_{2}$, $\mathrm{G} \operatorname{lnZ}(\mathrm{UMP})_{3}$, and unmodified forms. On the other hand, GInB had only 2 forms probably corresponding to the unmodified and $\mathrm{GlnB}(\mathrm{UMP})_{3}$, consistent with the observation in vivo (10). The results suggest that the levels of glutamine define the levels of PII uridylylation in A. brasilense and, therefore, the cellular nitrogen status.

We have examined in vitro the uridylylation of GInB and GInZ carried out by the $A$. brasilense GInD protein. Purified A. brasilense $\mathrm{G} \ln B$ and $\mathrm{G} \ln Z$ proteins were uridylylated by purified GInD in a process dependent on ATP and 2oxoglutarate and glutamine inhibits uridylylation and promotes deuridylylation.

\section{Acknowledgments}

We are very grateful to Dr. Marshall Geoffrey Yates for a critical review of the manuscript. We are also very grateful to Roseli Prado, Valter A. Baura, Daniela Fojo Seixas, and Julieta Pie for technical assistance, and to Mariana S. Araujo for providing the plasmid pMSA-L1. All of them are from Universidade Federal do Paraná, Curitiba, PR, Brazil.
1996; 178: 4830-4838

9. Araujo LM, Monteiro RA, Souza EM, Steffens MB, Rigo LU, Pedrosa FO, et al. GlnB is specifically required for Azospirillum brasilense NifA activity in Escherichia coli. Res Microbiol 2004; 155: 491-495.

10. Huergo LF, Chubatsu LS, Souza EM, Pedrosa FO, Steffens $M B$, Merrick M. Interactions between PII proteins and the nitrogenase regulatory enzymes DraT and DraG in Azospirillum brasilense. FEBS Lett 2006; 580: 5232-5236.

11. Huergo LF, Souza EM, Araujo MS, Pedrosa FO, Chubatsu LS, Steffens MB, et al. ADP-ribosylation of dinitrogenase reductase in Azospirillum brasilense is regulated by AmtBdependent membrane sequestration of DraG. Mol Microbiol 2006; 59: 326-337.

12. Araujo MS, Baura VA, Souza EM, Benelli EM, Rigo LU, Steffens MB, et al. In vitro uridylylation of the Azospirillum brasilense N-signal transducing GInZ protein. Protein Expr Purif 2004; 33: 19-24.

13. Forchhammer K, Hedler A, Strobel H, Weiss V. Heterotrimerization of PII-like signalling proteins: implications for PIImediated signal transduction systems. Mol Microbiol 1999; 33: $338-349$.

14. Son HS, Rhee SG. Cascade control of Escherichia coli glutamine synthetase. Purification and properties of PII protein and nucleotide sequence of its structural gene. J Biol Chem 1987; 262: 8690-8695.

15. Huergo LF, Merrick M, Pedrosa FO, Chubatsu LS, Araujo 
LM, Souza EM. Ternary complex formation between AmtB, GInZ and the nitrogenase regulatory enzyme DraG reveals a novel facet of nitrogen regulation in bacteria. Mol Microbiol 2007; 66: 1523-1535.

16. Gruswitz F, O'Connell J III, Stroud RM. Inhibitory complex of the transmembrane ammonia channel, AmtB, and the cytosolic regulatory protein, GInK, at 1.96 A. Proc Natl Acad Sci U S A 2007; 104: 42-47.

17. Conroy MJ, Durand A, Lupo D, Li XD, Bullough PA, Winkler FK, et al. The crystal structure of the Escherichia coli AmtBGInK complex reveals how GlnK regulates the ammonia channel. Proc Natl Acad Sci U S A 2007; 104: 1213-1218.
18. Senior PJ. Regulation of nitrogen metabolism in Escherichia coli and Klebsiella aerogenes: studies with the continuous-culture technique. J Bacteriol 1975; 123: 407-418.

19. Xu Y, Carr PD, Huber T, Vasudevan SG, Ollis DL. The structure of the PII-ATP complex. Eur J Biochem 2001; 268: 2028-2037.

20. Jiang P, Peliska JA, Ninfa AJ. Enzymological characterization of the signal-transducing uridylyltransferase/uridylyl-removing enzyme (EC 2.7.7.59) of Escherichia coli and its interaction with the PII protein. Biochemistry 1998; 37: 12782-12794. 